\title{
THE LAW OF MOMENTUM CONSERVATION AND SOME PROBLEMS OF METAGALACTIC ASTRONOMY
}

\author{
I. S. SHKLOVSKY \\ Sternberg Astronomical Institute, Moscow, U.S.S.R.
}

\begin{abstract}
Because of the anisotropic character of the generation of relativistic particles and synchrotron emission of active galactic nuclei and QSS it should be expected that 'magnetoids', the plasma bodies which are responsible for such generation, will attain large momenta. Therefore, magnetoids should be pushed out of nuclei with high velocities. This mechanism can explain several puzzling effects which are observed in the Metagalaxy, e.g. the jet in NGC 4486, connections between some galaxies and QSS and the total positive energy of some clusters of galaxies.
\end{abstract}

During the past decade many facts were accumulated which evidently support a famous hypothesis advanced by V. A. Ambartsumian (1958) about ejection of large aggregates of matter from active galactic nuclei. According to the concept of V. A. Ambartsumian, the matter initially ejected by some explosion process from a galactic nucleus must be in some hypothetical superdense state and during some time evolve into galaxies. However, we do not agree with this concept because a consequent development of it would lead to the conclusion that the laws of 'usual' physics are not applicable to the description of the processes in galactic nuclei. It seems to us that today there is no reason for such a radical assumption.

In this report we will make an attempt to give a simple and natural explanation for some strange phenomena which were discovered in the Metagalaxy and which at first sight confirm the concept of V. A. Ambartsumian.

Let us summarize these facts (for details see Burbidge (1970)).

(1) V. A. Ambartsumian emphasized the fact that for galaxy clusters, as a rule, $E+\Omega>0$ so that they have positive total energy. In order to avoid this difficulty, many authors advanced the hypothesis that there are large amounts of unseen matter inside these clusters. However, these attempts were not successful. As a matter of fact, there do exist clusters of galaxies which are unstable expanding systems. The characteristic time of such an expansion is $10^{8}-10^{9} \mathrm{yr}$, i.e. much less than the age of the Universe. From this fact follows inevitably the conclusion that the process of galaxy formation continues in our epoch. According to the point of view of V. A. Ambartsumian, galaxies are formed from the superdense matter ejected from active nuclei with high velocity. This hypothesis formally explains the observed total positive energy of a galactic cluster.

(2) There are chains and small groups of galaxies, e.g. Stefan's Quintet, Seyfert's Sextet, the Chain VV172, etc., in which one of the systems has an abnormally high velocity.

(3) Arp drew attention to the remarkable connection between the distribution of some galaxies and QSS over the sky. It is possible that in some cases there is a genetic 
coupling between these objects. The impression was created that some QSS were 'shot out' from active nuclei.

Note that this circumstance cannot be considered as an argument in favor of the 'local' hypothesis of the origin of QSS. The observed very large red shifts should be explained presumably by the cosmological expansion while the peculiar velocities of QSS may be comparatively large.

(4) In addition to the above-mentioned arguments, we must add another one. This argument concerns the so-called 'jets' of some galactic nuclei, notably the famous jet in NGC 4486. The assumption that this jet was formed by ejection of clouds of relativistic plasma from the nucleus of the galaxy leads to great difficulties. First, the time of synchrotron energy losses is unacceptably short. Moreover, within the framework of this hypothesis there is no explanation for the observed absence of expansion of knots in this jet. Note that almost exactly in the direction of the jet is situated the radiogalaxy NGC 4374. The alternative hypothesis for the production of the jet in NGC 4486 is the supposition of ejection in some direction of compact, gravitationally-bound aggregates of matter, which are permanently generating relativistic electrons. This supposition is free from the above-mentioned difficulties. We encounter similar problems in the interpretation of very compact details which are observed in some extended radio sources (e.g. Cyg A).

The analysis of the results of observation leads to the conclusion that along with ejection of adiabatic expanding clouds of relativistic and nonrelativistic plasma from active nuclei of galaxies there takes place the ejection of compact, non-expanding aggregates of matter which are in a state of very high activity (i.e. there is a powerful generation of relativistic particles). We will show that this process is a natural consequence of the law of momentum conservation and current ideas concerning the nature of activity of galactic nuclei and QSS.

Everybody is accustomed to the fact that during activity of galactic nuclei extremely large amounts of energy are liberated, up to $10^{61}-10^{62}$ ergs. This energy is generated mainly in the form of relativistic particles. But, as far as we know, until recently nobody drew attention to the fact that the relativistic particles and photons of synchrotron radiation take away a very large momentum from the region of galactic nuclei. The reason for this is the very anisotropic character of generation of relativistic particles by nuclei of galaxies. As a rule this generation occurs in two opposite directions. This follows from the observed duality of most radio sources. The photon synchrotron emission also must be anisotropic. This follows from the analysis of outbursts of radio emission from QSS and Seyfert nuclei. According to this analysis, the value of $H_{\perp}$ is comparatively small (Kellermann and Pauliny-Toth, 1968). On the other hand, the equipartition condition demands that the total $\mathrm{H}$ should be comparatively large. Consequently, $\mathrm{H}_{\perp} / \mathrm{H} \ll 1$ and synchrotron emission must be anisotropic.

This result is valid also for the very powerful infrared emission of QSS and Seyfert nuclei (Shklovsky, 1970).

Evidently, the total loss of momentum by a galactic nucleus caused by generation 
of relativistic particles and synchrotron quanta should be

$$
\Delta P=(2 \pi / c) \int W(\theta) \sin \theta \mathrm{d} \theta
$$

where $\theta$ is the angle between the axis of symmetry of the source and any direction and $W(\theta) \sin \theta d \theta$ denotes the energy which was emitted in this direction during the whole time of activity of the nucleus. If the generation of relativistic particles and photons by galactic nuclei were exactly axially symmetric, we should have $\Delta P=0$. However, in real conditions the case of exact axial symmetry does not exist. In any case the two components of a radio source are not similar in morphology and energy content. In some cases the two radio components and the 'parent' - the optically observed galaxy - are not situated along a straight line.

Consequently for our rough estimation we can assume, that

$$
\Delta P \approx W / c
$$

where $W$ denotes the total energy generated during the active phase of a nucleus. If $W \sim 10^{61} \mathrm{erg}, \Delta P \approx 3 \times 10^{50} \mathrm{~g} \mathrm{~cm} \mathrm{~s}^{-1}$.

According to the law of momentum conservation, the nucleus must obtain momentum $-\Delta P$ due to its energy generation. What are the consequences of this fact?

It seems to us that the most plausible hypothesis which explains the activity of QSS and galactic nuclei is the so-called 'magnetoid' hypothesis. According to this hypothesis, the source of activity of nuclei and QSS is connected with a large rotating magnetic plasma body or 'magnetoid'. Recently, Ozernoy (1971) undertook a very important and interesting development of this hypothesis.

It seems natural to suppose that the momentum $-\Delta P$ will be obtained by the magnetoid which is situated inside the nucleus of a galaxy. Denote the mass of magnetoid as $M$. Then we can write the following equations

$$
W=\alpha M c^{2},
$$

consequently

and

$$
\Delta P=\alpha M c=M v,
$$

$$
v=\alpha c
$$

where $\alpha \approx 1-10 \%$ denotes the efficiency of energy generation by the magnetoid. From this it follows that the 'back velocity' $v$ of the magnetoid must be in the range between several thousand and several tens of thousand $\mathrm{km} \mathrm{s}^{-1}$. This is adequate for the explanation of the above-mentioned phenomena.

The present situation in magnetoid theory does not allow us to describe its evolution in a precise way. Evidently such an evolution should be possible in different directions depending on the initial conditions (value of the mass, angular velocity and the law of rotation, etc.). It may be expected that in some cases the magnetoid during its evolution will be fragmented into many condensations which will evolve into stars 
while in other cases the magnetoid will collapse. It may be expected that the masses of magnetoids which were ejected from nuclei of galaxies will cover a wide range e.g. from $10^{3}$ to $10^{10} M_{\odot}$. It is most probable that this process will be recurrent: after ejecting one magnetoid from the nucleus of a galaxy another one will be formed which will evolve in a more or less similar way. It is reasonable to assume that the time interval between successive ejections of magnetoids is proportional to their masses. The cause of formation of magnetoids in nuclei of galaxies may be, for example, accretion of interstellar gas (Shklovsky, 1962) or disintegration of the stars caused by collisions or close encounters.

It is essential for both cases that nuclei of galaxies may be considered as very deep potential boxes.

Thus, it is possible to explain naturally all the strange phenomena mentioned above. Let us consider, for example, the jet in NGC 4486. Each condensation of this jet can be considered as a small magnetoid with a more or less regular magnetic field having a different orientation. These condensations are similar to small quasars. The absence of the emission lines in the spectrum of the jet can be explained by the violet shift. According to Equation (5), we may suppose that the velocities of these magnetoids are near to $\sim 10^{9} \mathrm{~cm} \mathrm{~s}^{-1}$.

Therefore, the age of condensations should be $\sim 10^{5} \mathrm{yr}$, while the mean interval between successive ejections of magnetoids from the nucleus of NGC 4486 must be several tens of thousands of years. From the total energy emitted by the jet during its lifetime $\left(\sim 3 \times 10^{42} \mathrm{erg}\right)$ we can roughly estimate the mass of the magnetoids as $10^{4} M_{\odot}$.

It is natural to expect (by analogy with quasars) that optical emission from the condensations of the jet may be variable. In addition we can expect variability of polarization from the condensations. Evidently the duration of activity of the nucleus of NGC 4486 may be much longer than $10^{5} \mathrm{yr}$.

Therefore, it may be expected that in NGC 4486 there will be many comparatively old magnetoids which are essentially less active than in the jet. It is not impossible that such objects may be identified with the so-called 'globular clusters' which are observed in NGC 4486. In (Shklovsky, 1968) we drew attention to a very strange phenomenon: most of the 'globular clusters' in NGC 4486 are situated in a quadrant with the axis coincident with the direction of the jet. In the case of 'true' globular clusters it is natural to expect spherical symmetry in their distribution.

Thus we see that the application of the law of momentum conservation to some of the problems of metagalactic astronomy will open new and far-reaching possibilities. In conclusion, we should emphasize that our attempt at analysis of this problem is a very rough and preliminary one, but further development of this idea may give new and interesting results.

\section{References}

Ambartsumian, V. A.: 1958, in Structure and Evolution of the Universe, Solvay Conference, Brussels, p. 241. 
Arp, H. C.: 1967, Astrophys. J. 148, 321.

Burbidge, G. R.: 1970, Ann. Rev. Astron. Astrophys. 8, 369.

Kellermann, K. I. and Pauliny-Toth, I.: 1968, Astrophys. J. Letters 152, L169.

Ozernoy, L. M.: 1971, this volume, p. 290.

Shklovsky, I. S.: 1962, Astr. Zh. 39, 591.

Shklovsky, I. S.: 1968, Astr. Zh. 45, 919.

Shklovsky, I. S.: 1970, Astr. Zh., in press. 